\title{
Mach-Zehnder interferometer implementation for thermo-optical and Kerr effect study
}

\author{
Arturs Bundulis*, Edgars Nitiss, Janis Busenbergs, Martins Rutkis \\ Institute of Solid State Physics, University of Latvia, Kengaraga 8, 1068 Riga, Latvia \\ *Corresponding author: arturs.bundulis@cfi.lu.lv
}

\begin{abstract}
In this paper, we propose the Mach-Zehnder interferometric method for third-order nonlinear optical and thermo-optical studies. Both effects manifest themselves as refractive index dependence on the incident light intensity and are widely employed for multiple opto-optical and thermo-optical applications. With the implemented method, we have measured the Kerr and thermo-optical coefficients of chloroform under CW, ns and ps laser irradiance. The application of lasers with different light wavelength, pulse duration and energy allowed us to distinguish the processes responsible for refractive index changes in the investigated solution. Presented setup was also used for demonstration of opto-optical switching. Results from Mach-Zehnder experiment were compared to Z-scan data obtained in our previous studies. Based on this, a quality comparison of both methods was assessed and advantages and disadvantages of each method were analyzed.
\end{abstract}

\section{Introduction}

Recently interest and demand for new materials with pronounced third-order nonlinear optical (NLO) properties have extensively grown. This has led to various new research trends structure-property relation studies [1-4], implementation of new experimental methods for NLO studies [5] as well as research for new practical applications of NLO materials [6]. The organic materials have attracted a great deal of attention due to the possibility of tuning their NLO properties by varying their molecular structure. Due to high third order response they are especially promising for application in optical data storage [7], optical power limiting [8] and optical data processing [9]. Although such opto-optical component prototypes have been presented in literature, materials with higher efficiencies are compulsory for their utilization in commercial devices. To advance in this research field it is essential to have a simple and accurate methods for measurement of NLO properties of different materials. In case of Kerr and two-photon absorption (TPA) NLO effects, experimental methods capable to detect small optical phase and optical power changes are needed.

Most of the experimental methods used to study third-order susceptibility associated with Kerr effect are based on measurement of the refractive index change induced by optical irradiance. For material possessing Kerr effect, refractive index can be described by the following equation:

$$
\boldsymbol{n}=\boldsymbol{n}_{\mathbf{0}}+\boldsymbol{n}_{\mathbf{2}} \cdot \boldsymbol{I},
$$

where $n_{0}$ is the linear refractive index, $n_{2}$ in nonlinear refractive index and $I$ is the incident light intensity. The real part of thirdorder susceptibility can then be calculated from nonlinear refractive index [10]. As shown in our previous paper, in order to correctly evaluate the magnitude of Kerr effect, it is important to consider different thermal processes that can also influence refractive index [3]. To avoid these effects, depending on absorption, thermal diffusivity and other fluid characteristics, it is important to choose correct laser operation parameters. In case of solutions, refractive index variations can be induced by two different thermal mechanisms - thermal diffusion and acoustic effects. The former effect is related to density fluctuations induced by sample heating while the latter effect is associated with acoustic wave induced across the waist of laser beam. Influence of these effects can be minimized by varying certain laser operation parameters. For diffusion effect, we need to decrease the amount of accumulated heat due to sequential laser pulses. This can be done by decreasing laser pulse repetition rate. The threshold frequency $v_{D}$ for diffusion effect is defined as:

$$
v_{D}=\frac{4 D}{w_{0}^{2}}
$$

where $D$ is the thermal diffusivity and $w_{0}$ is laser beam radius. In case of acoustic effect, it is crucial that laser pulse is long enough for an acoustic wave to propagate across laser beam waist. This gives us a pulse duration value at which acoustic effects become notable:

$$
t_{s}=\frac{w_{0}}{v_{s}}
$$

where $v_{s}$ is the speed of sound in fluid. Acoustic effects can be

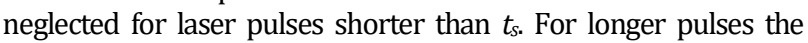
sample may heat up to reach the thermal equilibrium. By solving heat-transport equation we can conclude that time necessary for sample to reach thermal equilibrium is equal to [11]:

$$
t_{l} \approx \frac{\rho_{0} C w_{0}^{2}}{k}
$$

where $\rho_{0} C$ is the heat capacity per volume unit and $k$ is the thermal conductivity. All lasers with longer pulse duration than $t_{l}$ can be treated as continues-wave (CW) lasers in the Kerr coefficient measurement. The thermally induced refractive index changes 
caused by a CW light source can be calculated by using following equation:

$$
n_{2}=\left(\frac{d n}{d T}\right) \frac{\alpha w_{0}^{2}}{k}
$$

where $d n / d T$ characterizes refractive index changes due to temperature variations and $\alpha$ is linear absorption.

Several experimental methods have been implemented to study third-order NLO properties - Z-scan [12,13], nonlinear ellipse rotation [14], degenerated four-wave-mixing [15-17] and others. In clear majority of papers discussing third order NLO properties of materials, the Z-scan method is applied due to the simple experimental setup as well as possibility to perform simultaneous TPA and Kerr effect coefficient measurements. In this paper we describe our efforts to implement Mach-Zehnder interferometric (MZI) method [18] for determination of Kerr coefficient of solvents and solutions. MZI method is a two beam interferometric method that can be used for measurement of small optical path length variations. Due to its high sensitivity it is one of the most widely used methods to study second-order NLO properties [19] as well as for demonstration of electro-optical[20] and opto-optical modulations [21]. Compared to previous cases of MZI implementation [18,22] for third-order NLO studies, we have introduced an altered experimental setup as well as carried out experiments with two different wavelength lasers to demonstrate optical switching.

Nowadays scientists use wide spectrum of different lasers to study nonlinear optical responses, but the origins of nonlinear refractive index variations differ from wavelength to wavelength. In order to emphasize the importance of characteristics of light source employed in the experiment we compared different refractive index change inducing effects. Here we used an MZI experimental setup to determine third-order NLO properties for chloroform employing lasers widely used in experiments described in literature - continuous-wave (CW) [23], ns [24] and ps [25] lasers.

\section{Experimental Method}

The refractive index variations can be probed using an interferometric setup. To do this we implemented the MZI method as depicted in Fig. 1. Here a laser beam is split in reference beam $I_{1}$ and sample beam $I_{2}$ using a beam splitter (BS). We used a halfwavelength plate for $1064 \mathrm{~nm}$ and a Glan-Taylor prism to adjust the input laser power $I_{\text {in. }}$ Additionally, we recorded $I_{\text {in }}$ to know the values for $I_{1}$ and $I_{2}$. At the output of MZI the optical interference effect can be observed, and assuming negligible or no contribution from TPA effect the intensity Iout on the phase difference of sample and reference beams can be written as:

$$
I_{\text {out }}=\frac{I_{1}+I_{2}+2 \cdot \sqrt{I_{1} \cdot I_{2}} \cdot \cos \left(\Delta \varphi\left(I_{1}\right)+\varphi_{0}\right)}{2},
$$

where $I_{1}$ and $I_{2}$ are reference beam power and sample beam power, $\phi_{0}$ is the constant phase difference between both laser beams and $\Delta \phi\left(I_{1}\right)$ is the phase difference induced by the Kerr effect.

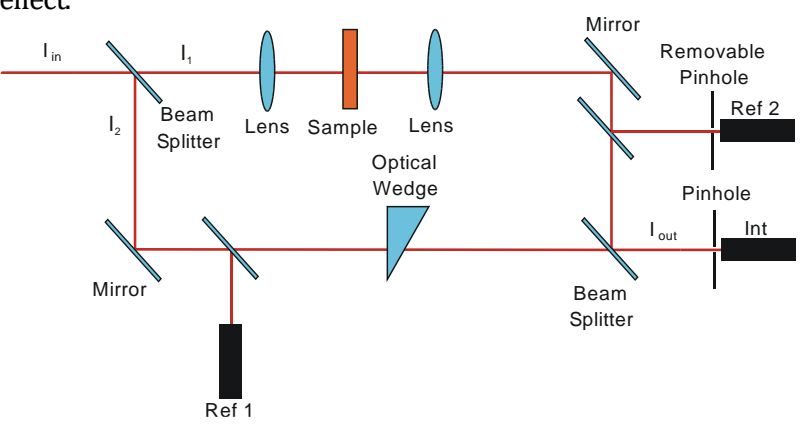

Fig. 1. MZI experimental setup using single laser beam.
We would like to highlight that for general case the beam power in the sample and reference arm in eq(6) are laser power dependent. Also, it is important to emphasize that unlike for Z-scan method the MZI analytical model is not limited to small phase changes $(\Delta \varphi<\pi)$. We placed two lenses with focal length of $\mathrm{f}_{1}=7 \mathrm{~cm}$ and $\mathrm{f}_{2}=8 \mathrm{~cm}$ in sample beam path to focus and then to collimate laser beam. A sample possessing Kerr effect can be described as a thin lens whose focal length depends on laser irradiation. This means that any positional deviation from focal point will lead to sample focusing or defocusing the laser beam. Therefore it is essential that sample is placed in focal point, so that phase changes would only lead to variations in optical path and not cause any changes in beam size. Moving sample holder was introduced in setup, so we could position the sample precisely in focal point. For characterizing the phase change induced by a focused Gaussian beam we used the following equations:

$$
\Delta \varphi=k n_{2} L_{e f f} I_{1}=\Delta \varphi^{*} I_{1},
$$

where $k$ is wave number and $L_{e f f}$ is effective length of sample:

$$
L_{e f f}=\frac{1-e^{-\alpha L}}{\alpha},
$$

where $\alpha$ is linear absorption and $L$ is sample length. Optical wedge was implemented in the setup to alter $\varphi_{0}$ and allows us to determine the sign for Kerr effect coefficient. During experimental measurements, we filtered out only the central part of interference using a small pinhole (diameter $d=1 \mathrm{~mm}$ ) and with main detector (Int) MZI output power was detected as function of laser power. Additionally, two reference detectors were introduced in setup. First reference detector (Ref $\mathbf{1}$ ) was used to monitor any external laser power variations present during measurement. Second reference detector (Ref 2) was used for two purposes: i) while a small pinhole (diameter $d=1 \mathrm{~mm}$ ) was inserted before this detector we could determine whether any beam size variations were present during measurement, ii) with no pinhole in front of the detector we measured full power of laser beam to detect any power changes due to TPA effect or any other absorption effect. To describe the TPA effect we can use Z-scan analytical expression assuming that sample is placed in focal point and instead of changing its position, we change laser beam power:

$$
T\left(I_{1}\right)=\sum_{m=0}^{\infty} \frac{\left(-\alpha_{2} L_{e f f}\right)^{m}}{(m+1)^{3 / 2}} I_{1}^{m} .
$$

If TPA absorption is not present, we can assume that $I_{1} \sim I_{i n}$.

Experimental measurements were also carried out using two different laser sources to study opto-optical switching - inducing refractive changes with one laser and probing them with other. In this case, we added optical filters before detectors to separate probing beam from both laser beams.

In our experiments, we studied refractive index changes of chloroform as it has been widely studied in literature and is a suitable reference material to compare MZI to Z-scan method. Samples were contained in a $2 \mathrm{~mm}$ thick photometric quartz cells. In our measurements we used the following lasers: i) $1064 \mathrm{~nm}$ laser with $8 \mathrm{~ns}$ pulse duration and $400-40000 \mathrm{~Hz}$ pulse repetition rates (EKSPLA NL 640), ii) $1064 \mathrm{~nm}$ laser with 30 ps duration and $10 \mathrm{~Hz}$ pulse repetition rate (EKSPLA SL 312), iii) 437, 632.8 and $780 \mathrm{~nm}$ CW lasers. We also employed MZI setup using different lasers for inducing and probing NLO effects. Particularly a 1064 $\mathrm{nm}$ Nd:YAG laser with 8 ns pulse duration (EKSPLA NL 640) was used as inducing beam and for probing three different CW lasers $437 \mathrm{~nm}, 632.8 \mathrm{~nm}$ and $780 \mathrm{~nm}$ were used. 


\section{Results and discussion}

\section{A. SINGLE LASER SETUP}

As will be shown later one of the main advantages of using MZI is the simplicity of experimental data analysis using eq (6). This of course is true for cases when only the laser beam phase is changed. Any external laser power fluctuations or beam size variations due to sample focusing or defocusing laser beam will complicate data fitting process. While external power fluctuations can be easily separated by recording reference beam power (Ref 1), beam size variation is related to sample positioning relative to focal point. To assume that sample will not change the beam size it must be placed in the focal point. We verified this by confirming a linear dependence between sample beam and input beam power using Ref 2 detector.

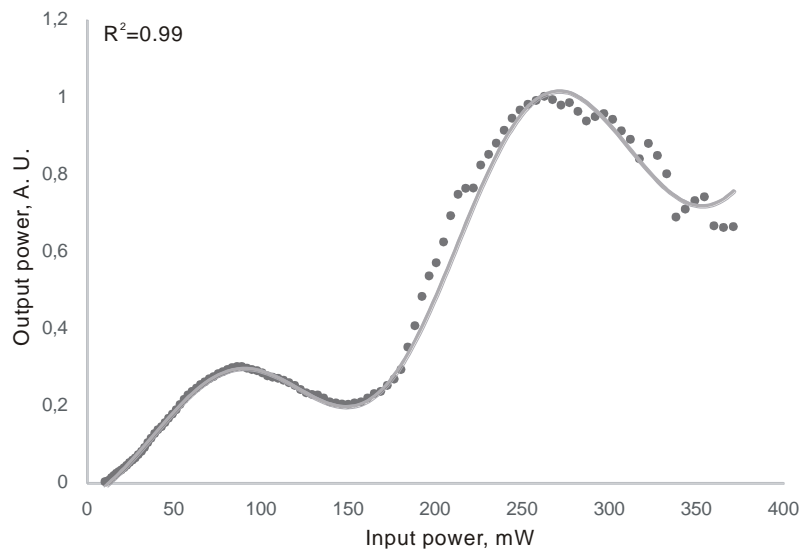

Fig. 2. Experimental measurement acquired with first MZI setup. Measured phase variation was of order $4 \pi$.

For single beam setup, refractive index changes were induced and detected with the same laser. This method allowed us to compare MZI to Z-scan method as both methods implements only one laser. For this evaluation to be acceptable, we employed the same laser as used in our Z-scan measurements. Experimental MZI measurements were carried out using a $1064 \mathrm{~nm}$ Nd:YAG laser with 8 ns pulse duration at pulse repetition frequency from 400 to $40000 \mathrm{~Hz}$. We measured nonlinear refractive index values for chloroform, as they have been previously measured using Z-scan method. Experimental measurement data are shown in Fig. 2 where data are fitted with simplified form of eq. (6). For our setup we can assume that $I_{1} / I_{2}=C$, where $C$ is a constant that characterizes BS. This allows us to rewrite eq. (6) in form:

$$
\begin{aligned}
& I_{\text {out }}=\frac{I_{1}+C \cdot I_{1}+2 \cdot \sqrt{I_{1} \cdot C \cdot I_{1}} \cdot \cos \left(\Delta \varphi\left(I_{1}\right)+\varphi_{0}\right)}{2}=\left(\frac{1+C}{2}\right) \cdot I_{1} \\
& \left(1+\left(\frac{2 \cdot \sqrt{C}}{1+C}\right) \cdot \cos \left(\Delta \varphi^{*} \cdot I_{1}+\varphi_{0}\right)\right)=A_{1} \cdot I_{1} \\
& \left(1+A_{2} \cdot \cos \left(\Delta \varphi^{*} \cdot I_{1}+\varphi_{0}\right)\right)
\end{aligned}
$$

where $I_{1}$ is laser power in sample beam, $A_{1}$ and $A_{2}$ in fitting constants that contains all constant variables and $\Delta \phi^{*}$ is phase variance component that is independent on laser power. Experimental data showed good correlation with analytical expression with $\mathrm{R}^{2}=0.99$. Nonlinear refractive index as function from pulse repetition frequency is shown in Fig. 3. Values varied from $-0.0185 \cdot 10^{-16} \mathrm{~m}^{2} / \mathrm{W}$ to $-3.24 \cdot 10^{-16} \mathrm{~m}^{2} / \mathrm{W}$. Additionally in Fig. 3. we included experimental data acquired with Z-scan method presented in our previous work. As it can be seen both methods produced similar results which lead us to believe that our MZI setup and data treatment produces correct results for nonlinear refractive index.

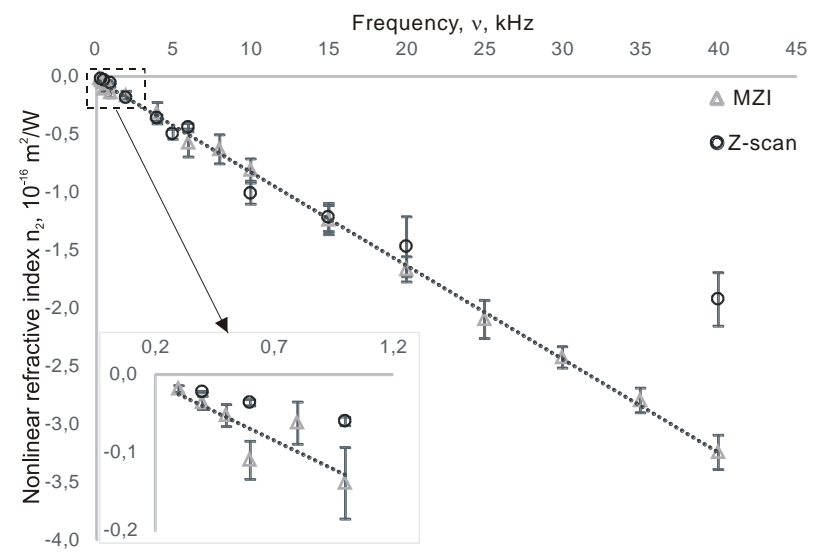

Fig. 3. Nonlinear refractive index as function of pulse repetition frequency. Z-scan data were taken from reference [3].

To further study thermo-optical properties of chloroform, we used CW laser operating $437 \mathrm{~nm}$. Experimental data are shown in Fig. 4. Acquired value for nonlinear refractive index was $n_{2}(437 \mathrm{~nm})=-$ $5.06 \pm 0.32 \cdot 10^{-12} \mathrm{~m}^{2} / \mathrm{W}$. As under CW laser irradiance sample reaches thermal equilibrium acquired value should agree with eq. (5). We used values of thermal conductivity and $d n / d T$ for chloroform presented in literature $[26,27]$ to calculate the thermo-

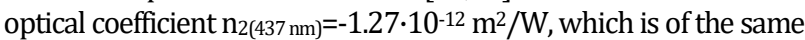
order as experimental value.

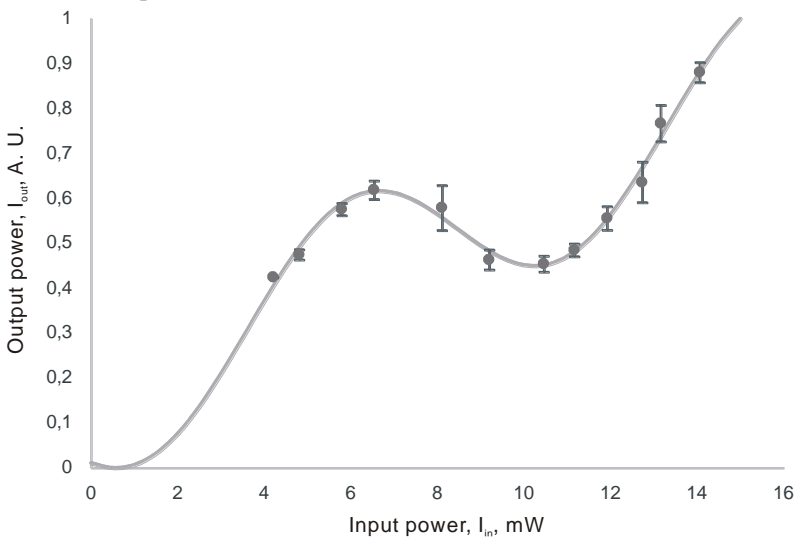

Fig. 4. MZI measurement with CW laser operating at $437 \mathrm{~nm}$.

To study Kerr effect of chloroform, we carried out measurements using a $1064 \mathrm{~nm}$ Nd:YAG laser with 30 ps pulse duration and $10 \mathrm{~Hz}$ pulse repetition rate. During experimental measurements, we encountered difficulties to acquire a stable interference pattern while using short pulse lasers. In spite of that we managed to measure nonlinear refractive index induced by Kerr effect and acquired value for chloroform $n_{2}=2.09 \pm 0.18 \cdot 10^{-15}$ $\mathrm{cm}^{2} / \mathrm{W}$ (experimental data can be seen in Fig. 5). This value is similar to the one acquired with Z-scan method $[3,25]$. Analytical expression showed good correlation with experimental data having $\mathrm{R}^{2}=0.95$. It is important to highlight that MZI becomes more difficult to implement for shorter pulse lasers due to difficulties to acquire stable interference pattern. As pulse duration becomes smaller, lasers coherence length becomes shorter. This leads to higher requirements for experimental setup as the optical path difference between both beams in MZI setup cannot exceed coherence length. 


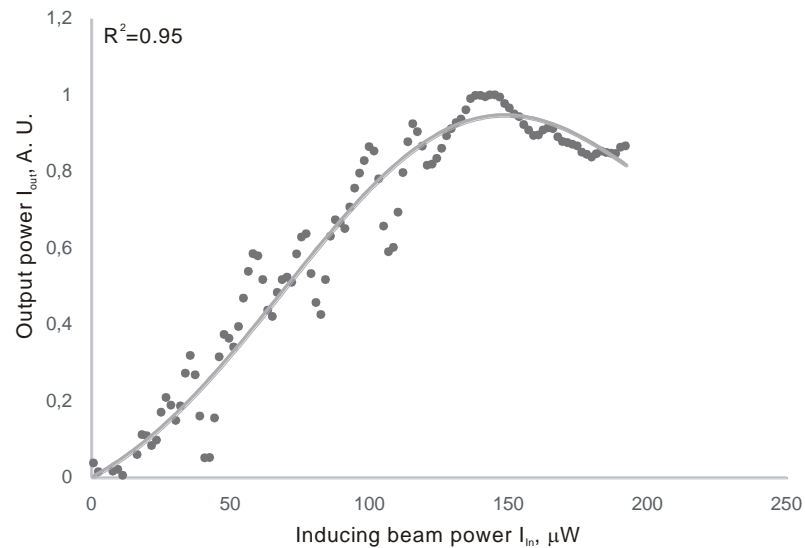

Fig. 5. Experimental data for measurement with $1064 \mathrm{~nm}$ Nd:YAG laser with $30 \mathrm{ps}$ pulse duration and $10 \mathrm{~Hz}$ repetition rate.

\section{B. TWO LASER SETUP}

The second setup (shown in Fig 6.) employs two lasers - one to induce refractive index changes and another one to probe them. During measurements probing beam power was kept constant while inducing beam power was altered. This setup allows us to demonstrate how MZI can be used for optical switching.

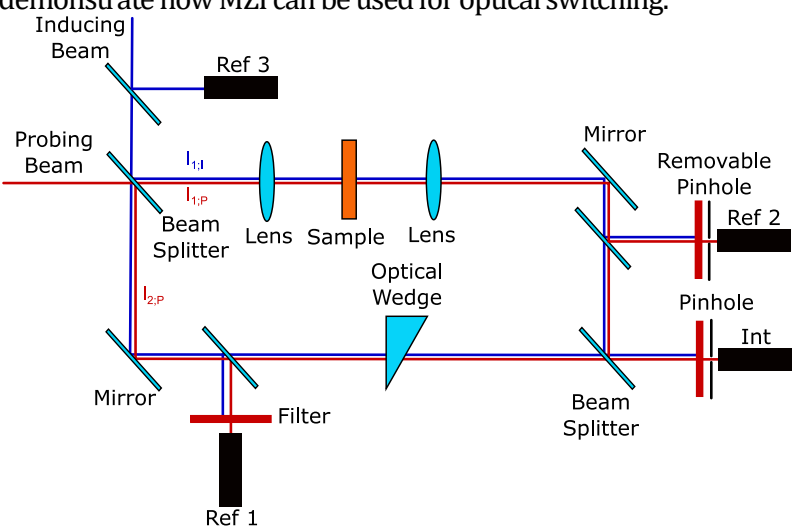

Fig. 6. MZI experimental setup using two lasers: inducing beam, which changes refractive index of sample, and probing beam with which we measure the refractive index variance.

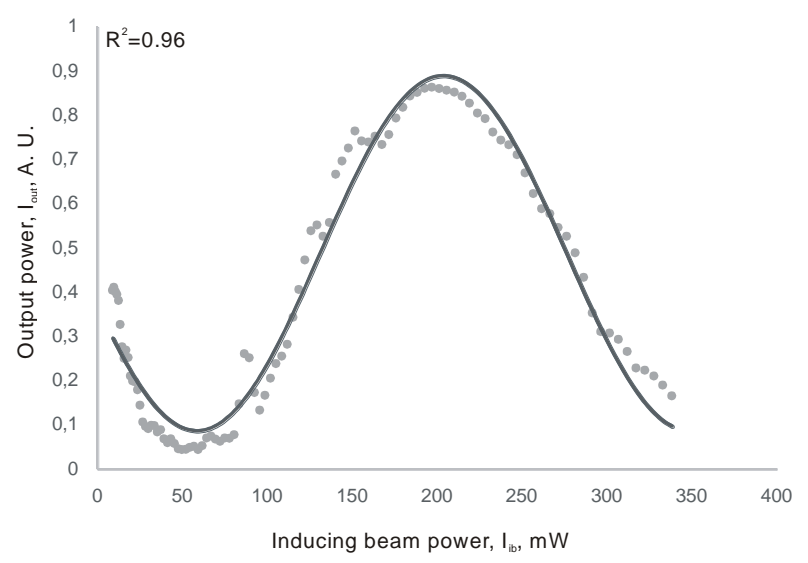

Fig. 7. Probing beam output power as function of Inducing beam power.

In second MZI setup we induced refractive index changes with $1064 \mathrm{~nm}$ Nd:YAG laser with 8-ns pulse duration and probed the changes with CW laser with different wavelength. Experimental data for $780 \mathrm{~nm}$ probing laser while inducing Nd:YAG laser was set on $30 \mathrm{kHz}$ pulse repetition rate is shown in Fig 7. For data fitting we use a different form of eq (6):

$$
I_{\text {out }}=\frac{I_{1 ; P}+I_{2 ; P}+2 \cdot \sqrt{I_{1 ; P} \cdot I_{2 ; P}} \cdot \cos \left(\Delta \varphi\left(I_{1 ; P}+I_{1 ; I}\right)+\varphi_{0}\right)}{2},
$$

where $I_{1 ; P}$ and $I_{2 ; P}$ are sample and reference beam power for probing laser and $\mathrm{I}_{1 ; \mathrm{i}}$ is sample beam for inducing laser. In this case $\mathrm{I}_{1 ; \mathrm{P}}$ and $\mathrm{I}_{2 ; \mathrm{P}}$ are constant which allows us to simplify this equation in form:

$$
\begin{gathered}
I_{\text {out }}=\frac{I_{1 ; P}+I_{2 ; P}+2 \cdot \sqrt{I_{1 ; P} \cdot I_{2 ; P}} \cdot \cos \left(\Delta \varphi\left(I_{1 ; P}+I_{1 ; I}\right)+\varphi_{0}\right)}{2}= \\
\frac{I_{1 ; P}+I_{2 ; P}}{2}+\sqrt{I_{1 ; P} \cdot I_{2 ; P}} \cdot \cos \left(\Delta \varphi^{*} \cdot I_{1 ; I}+\left(\Delta \varphi^{*} \cdot I_{1 ; I}\right.\right. \\
\left.\left.\varphi_{0}\right)\right)=B_{1}+B_{2} \cdot \cos \left(\Delta \varphi^{*} \cdot I_{1}+\varphi_{0}\right)
\end{gathered}
$$

where $B_{1}$ and $B_{2}$ are approximation constants that contains all constant parameters. Experimental data showed good correlation with analytical expression having $\mathrm{R}^{2}=0.96$. Using eq (7) and acquired values for $\Delta \phi^{*}$ we calculated that nonlinear refractive

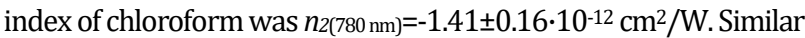
experiments were carried out with $437 \mathrm{~nm}$ and $632.8 \mathrm{~nm}$ probing lasers. Nonlinear refractive index values as functions of pulse repetition rate for all probing lasers as well as single beam experiments are shown in Fig 8. From experimental data, it is evident that the $n_{2}$ acquired for $437 \mathrm{~nm}$ is considerably larger than for other wavelengths. This shows that refractive index changes depend on probing laser beam. As probing laser beam wavelength is approaching absorption maximum refractive index change increases. Experimental results confirm that MZI can be used to study effects involving different laser sources for effect inducing and probing. As an example, the setup could be used for probing the third-order susceptibility $\chi\left(\omega_{1} ; \omega_{1}, \omega_{2},-\omega_{2}\right)$ at arbitrary optical frequencies $\omega_{1}$ and $\omega_{2}$.

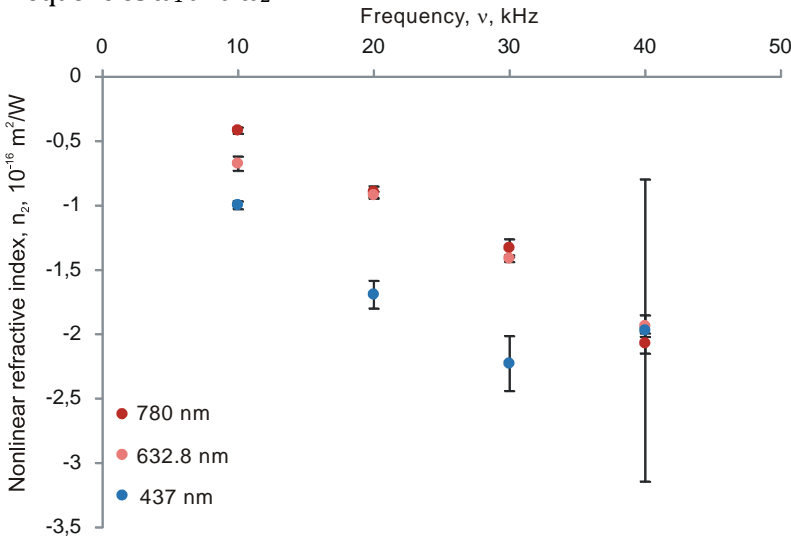

Fig. 8. Nonlinear refractive index values for different probing laser wavelengths.

By comparing experimental data for nonlinear refractive index acquired by MZI method with Z-scan data presented in literature, we can see that experimental values acquired by both methods are similar. This led us to believe that MZI can be used to correctly evaluate refractive index changes. MZI offers high sensitivity as well as simple data processing. By introducing an optical wedge, we can also determine the sign of nonlinear refractive index. We must acknowledge that for Z-scan method former process is much simpler. Also, it is hard to acquire stable interference pattern when using lasers with short pulse duration ( $t \approx 1 \mathrm{ps}$ ) due to very small coherence length. At the same time, there were no problems when using lasers with pulse duration at ns scale and can be easily implemented to study thermo-optical effects. The summary of all measurements presented in this work can be found in table 1 . 
Table 1. Nonlinear optical effects

\begin{tabular}{|c|c|c|}
\hline Laser & $\begin{array}{c}\text { Dominant } \\
\text { Effect }\end{array}$ & Value \\
\hline $\begin{array}{c}\mathrm{CW} \\
\text { laser }\end{array}$ & $\begin{array}{c}\text { Thermo- } \\
\text { optical effect }\end{array}$ & $-5.06 \pm 0.32 \cdot 10^{-12} \mathrm{~m}^{2} / \mathrm{W}$ \\
\hline $\begin{array}{c}\text { Ns } \\
\text { laser }\end{array}$ & $\begin{array}{c}\text { Thermo- } \\
\text { optical effect }\end{array}$ & $\begin{array}{c}\text { Depends on laser frequency and } \\
\text { pulse duration }(-0.0185 \ldots- \\
\left.3.24 \cdot 10^{-16} \mathrm{~m}^{2} / \mathrm{W}\right)\end{array}$ \\
\hline Ps laser & Kerr effect & $2.09 \pm 0.18 \cdot 10^{-15} \mathrm{~cm}^{2} / \mathrm{W}$ \\
\hline
\end{tabular}

\section{Conclusions}

To study thermo-optical and Kerr effect in chloroform we implemented MZI setup using three different lasers - picosecond, nanosecond and CW. Firstly we used CW laser to determine thermo-optical coefficient of chloroform. By comparing experiments results with evaluation of thermally induced refractive index changes, we concluded that experimental value is of the same order. This proves that refractive index changes are purely a thermal effect. Afterwards we studied thermo-optical effect dependence on pulse repetition rate using nanosecond laser. Acquired results were similar to ones reported in literature from Z-scan experiments. This was essential to prove that both methods - MZI and Z-scan - provide similar experimental results. Lastly, we used picosecond laser to determine Kerr coefficient of chloroform. Acquired value agreed with data presented in literature which indicates that this method can be used to study third-order NLO properties.

We compared MZI method to Z-scan to determine in which situations each of the methods is more convenient. Main drawback of MZI is the difficulty to achieve stable interference for short pulse lasers such as picosecond pulse lasers. This limitation can be avoided by using two laser MZI setup with CW or long pulse laser as probing laser. Otherwise it is more suitable to use Z-scan when working with short pulse lasers. At the same time, MZI method possesses distinct advantages such as simpler data processing and higher sensitivity, which makes it ideal for studying materials using nanosecond and longer pulse lasers as well as for different thermo-optical studies.

Funding Information. Scientific Research Project for Students and Young Researchers Nr. SJZ/2016/10; National Research Program "Multifunctional Materials and Composites, Photonics and Nanotechnology" (IMIS2) project "Photonics and materials for photonics".

\section{References}

1. L. A. Padilha, S. Webster, O. V Przhonska, H. Hu, D. Peceli, J. L. Rosch, M. V Bondar, A. O. Gerasov, Y. P. Kovtun, M. P. Shandura, A. D. Kachkovski, J. Hagan, and E. W. Van Stryland, "Nonlinear absorption in a series of Donor - p - Acceptor cyanines with different conjugation lengths $\dagger, "$ J. Mater. Chem. 19, 7503-7513 (2009).

2. S. Webster, J. Fu, L. A. Padilha, O. V Przhonska, D. J. Hagan, E. W. Van Stryland, M. V Bondar, Y. L. Slominsky, and A. D. Kachkovski, "Comparison of nonlinear absorption in three similar dyes: Polymethine , squaraine and tetraone," Chem. Phys. 348, 143-151 (2008).

3. A. Bundulis, E. Nitiss, I. Mihailovs, J. Busenbergs, and M. Rutkis, "Study of Structure-Third-Order Susceptibility Relation of Indandione Derivatives,"
J. Phys. Chem. C acs.jpcc.6b07003 (2016).

4. D. Gudeika, A. Bundulis, I. Mihailovs, D. Volyniuk, M. Rutkis, and J. V. Grazulevicius, "Donor and acceptor substituted triphenylamines exhibiting bipolar charge-transporting and NLO properties," Dye. Pigment. 140, 431-440 (2017).

5. X. Yan, Z. Liu, X. Zhang, W. Zhou, and J. Tian, "Polarization dependence of Z-scan measurement: theory and experiment," Opt. Express 17, 18211823 (2009).

6. A. E. Sifain, L. F. Tadesse, J. A. Bjorgaard, D. E. Chavez, O. V. Prezhdo, R. J. Scharff, and S. Tretiak, "Cooperative enhancement of the nonlinear optical response in conjugated energetic materials: A TDDFT study," J. Chem. Phys. 146, 114308 (2017).

7. D. Hu, Y. Hu, W. Huang, and Q. Zhang, "Two-photon induced data storage in hydrogen bonded supramolecular azopolymers," Opt. Commun. 285, 4941-4945 (2012).

8. A. A. Borshch, M. S. Brodyn, V. N. Starkov, V. I Rudenko, V. I. Volkov, A. Y. Boyarchuk, and A. V. Semenov, "Broadband optical limiting in thin nanostructured silicon carbide films and its nature," Opt. Commun. 364, 88-92 (2016).

9. S. Ahadi and N. Granpayeh, "Femtosecond alloptical switching based on asymmetric plasmonic Kerr Fabry-Perot resonators," Opt. Commun. 349, 36-41 (2015)

10. R. del Coso and J. Solis, "Relation between nonlinear refractive index and third-order susceptibility in absorbing media," J. Opt. Soc. Am. B 21, 640 (2004).

11. R. W. Boyd, "Nonlinear Optics," (2003).

12. M. S. Bahae, a a Said, T. H. Wei, D. J. Hagan, and E W. Van Stryland, "Sensitive Measurements of Optical Nonlinearities Using a Single Beam," IEEE J. Quantum Electron. 26, 760-769 (1990).

13. J. E. Aber, M. C. Newstein, and B. A. Garetz "Femtosecond optical Kerr effect measurements in silicate glasses," J. Opt. Soc. Am. B 17, 120-127 (2000).

14. Z. Liu, X. Yan, J. Tian, W. Zhou, and W. Zang, "Nonlinear ellipse rotation modified Z-scan measurements of third-order nonlinear," Opt. Express 15, 13351-13359 (2007).

15. I. V Kityk, A. Fahmi, B. Sahraoui, G. Rivoire, and I Feeks, "Nitrobenzene as a material for the fastrespond degenerate four-wave mixing," Opt. Mater. (Amst). 16, 417-429 (2001).

16. M. Samoc, A. Samoc, and B. Luther-davies, "Femtosecond Z-scan and degenerate four-wave mixing measurements of real and imaginary parts of the third-order nonlinearity of soluble conjugated polymers," J. Opt. Soc. Am. B 15, 817825 (1998). 
17. M. J. Bloemer, J. W. Haus, and P. R. Ashley, "Degenerate four-wave mixing in colloidal gold as a function of particle size," J. Opt. Soc. Am. B 7, 790795 (1990)

18. G. Boudebs, M. Chis, and X. N. Phu, "Third-order susceptibility measurement by a new MachZehnder interferometry technique," J. Opt. Soc. Am. B 18, 623 (2001).

19. E. Nitiss, A. Bundulis, A. Tokmakov, J. Busenbergs, E. Linina, and M. Rutkis, "Review and comparison of experimental techniques used for determination of thin film electro-optic coefficients," Phys. Status Solidi Appl. Mater. Sci. 212, 1867-1879 (2015).

$20 . \quad J$. Brosi, C. Koos, L. C. Andreani, J. Leuthold, and W. Freude, "High-speed low-voltage electro-optic modulator with a polymer-infiltrated silicon photonic crystal waveguide," 16, 180-185 (2008).

21. I. Glesk, P. J. Bock, P. Cheben, J. H. Schmid, J. Lapointe, and S. Janz, "All-optical switching using nonlinear subwavelength Mach-Zehnder on silicon," Opt. Express 19, 14031 (2011).

22. G. Boudebs, F. Sanchez, C. Duverger, and B. Boulard, "Improvement of Mach-Zehnder interferometry technique for third-order susceptibility measurement," Opt. Commun. 199, 257-265 (2001).
23. S. Jeyaram and T. Geethakrishnan, "Third-order nonlinear optical properties of acid green 25 dye by Z-scan method," Opt. Laser Technol. 89, 179185 (2017).

24. J. Yang, Y. Song, J. Gu, and H. Zheng, "Determinations of the transient thermal lensing effect in metal cluster Polymer \{WS4Cu4I2(bpe)3\}n solution by the use of the Z-scan," Opt. Commun. 282, 122-125 (2009).

25. T. Cassano, R. Tommasi, M. Ferrara, F. Babudri, G. M. Farinola, and F. Naso, "Substituent-dependence of the optical nonlinearities in poly(2,5-dialkoxy-pphenylenevinylene) polymers investigated by the Z-scan technique," Chem. Phys. 272, 111-118 (2001).

26. A. Samoc, "Dispersion of refractive properties of solvents: Chloroform, toluene, benzene, and carbon disulfide in ultraviolet, visible, and nearinfrared Dispersion of refractive properties of solvents: Chloroform, toluene, benzene, and carbon disulfide in," J. Appl. Phys. 6167, (2014).

27. H. Cabrera, A. Marcano, and Y. Castellanos, "Absorption coefficient of nearly transparent liquids measured using thermal lens spectrometry," Condens. Matter Phys. 9, 385-389 (2006).

Institute of Solid State Physics, University of Latvia as the Center of Excellence has received funding from the European Union's Horizon 2020 Framework Programme H2020-WIDESPREAD-01-2016-2017-TeamingPhase2 under grant agreement No. 739508, project CAMART² 